\title{
Essential and Non-Essential Amino Acids in Relation to Glutamate
}

\author{
Toru Takahashi ${ }^{1}$, Eri Toda ${ }^{2}$, Ram B. Singh ${ }^{3 *}$, Fabien De Meester $^{4}$, Agnieszka Wilczynska $^{4}$, \\ Douglas Wilson $^{5}$ and Lekh R. Juneja ${ }^{6}$
}

\author{
${ }^{I}$ Graduate School of Human Environment Science, Fukuoka Women's University, Japan \\ ${ }^{2}$ Tokai University Hachioji Hospital, Tokyo, Japan \\ ${ }^{3}$ Halberg Hospital and Research Institute, India \\ ${ }^{4}$ The Tsim Tsoum Institute, Krakow, Poland \\ ${ }^{5}$ School of Medicine and Health, Durham University, UK \\ ${ }^{6}$ Taiyo Kagaku, Yokkaichi, Japan
}

\begin{abstract}
The protein intake during the Palaeolithic period has been estimated to be two and a half fold greater than current recommendations. Hydrogen, nitrogen, oxygen and carbon atoms are important components of essential and nonessential amino acids which are components of proteins. These simple molecules play an important role in the human body. The 22 known amino acids, essential and nonessential, affect a broad range of physical and mental processes. Recent studies indicate that amino acids are cell signalling molecules as well as being regulators of gene expression and the protein phosphorylation cascade. The majority of the neurotransmitters is composed of amino acids and can influence biological functions related to brain-body interactions. Amino acids are key precursors for syntheses of hormones and low-molecular weight nitrogenous substances with each having enormous biological importance. Physiological concentrations of amino acids and their metabolites; nitric oxide, polyamines, glutathione, taurine, thyroid hormones, and serotonin are required for the biological functions in our body. These metabolites are known to be protective against cardiovascular diseases and degenerative diseases of the brain. However, increased concentrations of amino acids and their products (e.g., ammonia, homocysteine, and asymmetric dimethylarginine) are pathogenic factors for neurological disorders, oxidative stress and cardiovascular disease. Glutamate is a nonessential amino acid present in many foods that is considered protective against obesity and cardiovascular diseases. Therefore a balance between amino acids and other nutrients appears to be important for normal physiological functions without any adverse effects.
\end{abstract}

Keywords: Glutamic acid, monosodium glutamate, proteins, fatty acids.

\section{INTRODUCTION}

The diet in the Palaeolithic period was characterized with natural foods; fruits, vegetables, green vegetables, seeds, milk and eggs. Fish, meat from running animals and honey were also available to early man [1-4]. These foods were continued to be consumed by pre-agricultural humans which shaped modern human's genetic nutritional requirement. However, with the development of agriculture about 10,000 years ago, marked changes in the food supply have been observed. Despite changes in food intakes, only nonsignificant alteration in our genes have occurred over the past ten centuries due to presence of $\mathrm{w}-3$ fatty acids, amino acids, vitamins, and antioxidants in the diet [2-4], until recently. The spontaneous mutation rate for nuclear DNA is estimated at $0.5 \%$ per million years. Hence, over the past 10,000 years, there has been time for very little change in our genes, possibly $0.005 \%$ [2-4]. Our genes appear to be

*Address correspondence to this author at the Tsim Tsoum Institute, Ul. Golebia 2, 31-007 Krakow, Poland; Tel/Fax: 0091591 2417437;

E-mails icn2005@bsnl.in,rbs@tsimtsoum.net similar to the genes of our ancestors during the Palaeolithic period 40,000 years ago, the time when our genetic profile was established. It seems that now humans generally appear to live in a nutritional environment which completely differs from that for which our genetic constitution was selected. However, it is during the last 100-160 years that dietary intakes have changed significantly, causing increased intake of saturated fatty acids (SFA) and linoleic acid, and a decrease in w-3 fatty acids and amino acids from grain-fed cattle, tamed at farm houses, rather than meat from running animals (Tables 1-4).

\section{NUTRIENT INTAKE IN THE PALAEOLITHIC PERIOD}

The food and nutrient intake among hunter-gatherers and during the Palaeolithic period are given in Tables 1-3, (Fig. 1). There is a marked reduction in consumption of w-3 fatty acids, vitamins, antioxidants, and amino acids and a significant increase in the intakes of carbohydrates (mainly refined), fat (saturated, trans fat, and linoleic acid), and salt compared to the Palaeolithic period [1-4]. The protein intake was two and a half fold greater (33 vs. $13 \%$ ) in the Palaeo- 
Table 1. Food and Nutrient Intake among Hunter-Gatherer and Western Population

\begin{tabular}{|l|l|l|}
\hline Food and Nutrient & Hunter-Gatherer & Western Population \\
\hline \hline Energy density & Low & High \\
Protein & High & Low-moderate \\
Animal & High & Low -moderate \\
Vegetable & Very low & Low -moderate \\
Carbohydrate & Low-moderate(slowly absorbed) & Moderate-rapidly absorbed \\
Fiber & High & Low \\
Fat & Low & High \\
Animal & Low & High \\
Vegetable & Very low & High \\
Total w-3 & High(2.3g/day) & Low (0.2g/day) \\
Ratio w-6:w-3 & Low 2.4 & High $15-20$ \\
Vitamins and minerals & High & Low \\
Essential amino acids & High & Low \\
\hline
\end{tabular}

Modified from Eaton et al., ref 2,3,4.

lithic diet compared to modern diet which may be due to availability of amino acids in the evolutionary diet [5] (Table 3, Fig. 1). It is clear that the early diet was rich in essential and nonessential amino acids like glutamate which may have provided the major taste because salt and sugar were unknown during Palaeolithic period. According to old Sanskrit

Table 2. Estimated Fatty Acid Consumption in the Late Palaeolithic Period

\begin{tabular}{|l|l|}
\hline Sources & $\begin{array}{l}\text { Fatty Acids(g/day) } \\
\text { en 35.65/day }\end{array}$ \\
\hline \hline Plants & \\
Linoleic acid & 4.28 \\
Alpha-linoleic acid & 11.40 \\
Animal & \\
Linoleic acids & 4.56 \\
Alpha-linolenic acid & 1.21 \\
Total & \\
Linoleic acid & 8.84 \\
Alpha linolenic acid & 12.60 \\
Animal & \\
Arachidonic acid(w-6) (AA) & 1.81 \\
Eicosapentaenoic acid(w-3)(EPA) & 0.39 \\
Docosatetraenoic acid(w-6) (DTA) & 0.12 \\
Docosapentaenoic acid(w-3)(DPA) & 0.42 \\
Docosahexaenoic acid(w-3)(DHA) & 0.27 \\
Ratios of w-6/w-3 & \\
Linoleic acid/alpha linolenic acid & 0.70 \\
AA+DTA/EPA+DPA+DHA & 1.79 \\
Total w-6/w-3 & 0.79 \\
\hline
\end{tabular}

Modified from Eaton et al., Ref 2-4.

Energy intake (en) based on 35:65 animal: plant sources. proverb, there are four classic tastes; sweet, salty, sour, and bitter, of which salty appears to be the greatest and of most recent origin. The fifth taste discovered by K Ikeda in 1908 (umami taste) appears to be the natural taste of the foods available to early man such as human milk, fish, chicken, sea foods, sea weed, all rich in glutamate providing the umami taste (Table 4) $[6,7]$. It may be proposed that the higher risk of cardiovascular diseases and diabetes among people of South Asian origin living anywhere in the world, compared to indigenous populations and other Asian populations, may be due to lower intake of glutamate among South Asians compared to other populations. This poses the possibility that glutamate may have beneficial effects on central obesity and metabolic syndrome and can modulate mind-body interactions, because recent research indicate that glutamate can prevent obesity in mice and has beneficial effects on the hypothalamus, hippocampus and amygdala. Glutamate is also involved in the activation of ATP-sensitive $\mathrm{K}+$ (KATP) channels by $\mathrm{H}_{2} \mathrm{O}_{2}$ and glutamate-dependent inhibition of striatal dopamine release [8].

\section{EFFECTS OF DIET ON RISK OF CARDIOVASCU- LAR DISEASE}

Western diets are characterized by high omega- 6 and low omega-3 fatty acid intake, whereas during the Palaeolithic period when human's genetic profiles were being established, there was a balance between omega- 6 and omega- 3 fatty acids as well as amino acids [1-3, 9-16]. Therefore, humans today live in a nutritional environment that differs from that for which our genetic constitution was selected. Cohort studies have demonstrated that European vegetarian diets of Seventh-Day Adventists, Mediterranean diet (fruit, vegetables, nuts, whole grains, olive oil, wine fish, chicken), Japanese diets; (vegetables, raw fish, whole rice), IndoMediterranean diet(whole grains, fruits, vegetables, nuts and mustard oil) and the French paradox diet ( vegetables and wine) are protective against cardiovascular disease, diabetes and cancer. All these diets are rich in amino acids as well as 
Table 3. Nutrient Composition in the Late Palaeolithic and Current Recommendations

\begin{tabular}{|l|l|l|}
\hline Nutrient & Late Palaeolithic & Current Recommendation \\
\hline \hline Total dietary energy\% & & \\
Protein & 33 & 12 \\
Carbohydrate & 46 & 58 \\
Fat & 21 & 30 \\
Alcohol & -0 & moderate alcohol \\
P/S ratio & 1.41 & 1.00 \\
Cholesterol,mg & 520 & 300 \\
Fiber,g & $100-150$ & $30-60$ \\
Sodium, mg & 690 & $1100-3300$ \\
Calcium,mg & $1500-2000$ & $800-1600$ \\
Ascorbic acid,mg & 440 & 60 \\
W-6/W-3 ratio & $1: 1$ & $1: 5$ \\
\hline
\end{tabular}

Modified from Eaton et al., ref 2,3,4.

w-3 fatty acids, however, only omega-3 fatty acids has been considered in the mechanism of benefit without any consideration for amino acids. The total energy from proteins may be approximately $16 \%$ en per day which is closer to huntergatherers diet.

\section{AMINO ACIDS}

Essential and nonessential amino acids consist of hydrogen, nitrogen, oxygen and carbon atoms. These simple molecules play an important role in the human body [5, 1719]. The 22 known amino acids, essential and nonessential, affect a broad range of physical and mental processes. Recent studies have witnessed the discovery that amino acids are cell signalling molecules as well as being regulators of gene expression and the protein phosphorylation cascade [5]. Majority of the neurotransmitters can influence mind-body interactions and are composed of amino acids. Additionally, amino acids are key precursors for syntheses of hormones and low-molecular weight nitrogenous substances with each having enormous biological importance. Physiological

Table 4. Evolutionary Foods Rich in Glutamate

\begin{tabular}{|c|c|}
\hline Nonvegetarian & Vegetarian \\
\hline $\begin{array}{ll}\text { - } & \text { Beef and } \\
\text { - } & \text { chicken } \\
\text { - } & \text { Fish } \\
\text { - } & \text { Cheese } \\
\text { - } & \text { Human breast milk } \\
\text { - } & \text { Sea foods; } \\
\text { - } & \text { crabs, scallop }\end{array}$ & $\begin{array}{l}\text { - } \text { Soyabeans and beans } \\
\text { - } \text { Corn } \\
\text { - } \text { green peas } \\
\text { - } \text { Tomato } \\
\text { - } \text { spinach } \\
\text { - } \text { cabbage } \\
\text { - } \text { mushroom } \\
\text { - } \text { onion } \\
\text { - Sea weeds } \\
\text { - dried lever } \\
\text { - } \text { kelp(konbu) }\end{array}$ \\
\hline
\end{tabular}

concentrations of amino acids and their metabolites (e.g., nitric oxide, polyamines, glutathione, taurine, thyroid hormones, and serotonin) are required for the biological functions in our body. However, elevated levels of amino acids and their products (e.g., ammonia, homocysteine, and asymmetric dimethylarginine) are pathogenic factors for neurological disorders, oxidative stress, and cardiovascular disease. Thus, an optimal balance among amino acids in the diet and circulation is crucial for whole body homeostasis. There is growing recognition that besides their role as building blocks of proteins and polypeptides, some amino acids regulate key metabolic pathways that are necessary for maintenance, growth, reproduction, and immunity. They are called functional amino acids, which include arginine, cysteine, glutamine, leucine, proline, and tryptophan. Dietary supplementation with one or a mixture of these amino acids may be beneficial [5, 17-19] for ameliorating health problems at various stages of the life cycle (e.g., fetal growth restriction, neonatal morbidity and mortality, weaningassociated intestinal dysfunction and wasting syndrome, obesity, diabetes, cardiovascular disease, the metabolic syndrome, and infertility) [5]. Amino acids can also optimize efficiency of metabolic transformations to enhance muscle growth, milk production, egg and meat quality and athletic performance, while preventing excess fat deposition and reducing adiposity. Thus, amino acids have important functions in both nutrition and health.

Branched-chain amino acids may have psychological effects as well. Athletes often neglect the importance of mental "toughness," but fatigue can increase errors of omission and commission. According to a review, cognitive performance suffers during physical exercise [19]. There is evidence that branched-chain amino acids improved mental performance and decrease fatigue during a boating competition [5]. Non-essential amino acids produce similar effects on human physiology. Ingesting ornithine alphaketoglutarate increases circulating levels of the non-essential amino acid glutamine which may increase the body size of undersized children without glutamine toxicity. Other nonessential amino acids also have physiological effects. 


\section{The Tsim Tsoum Concept \& Evolutionary Diet}

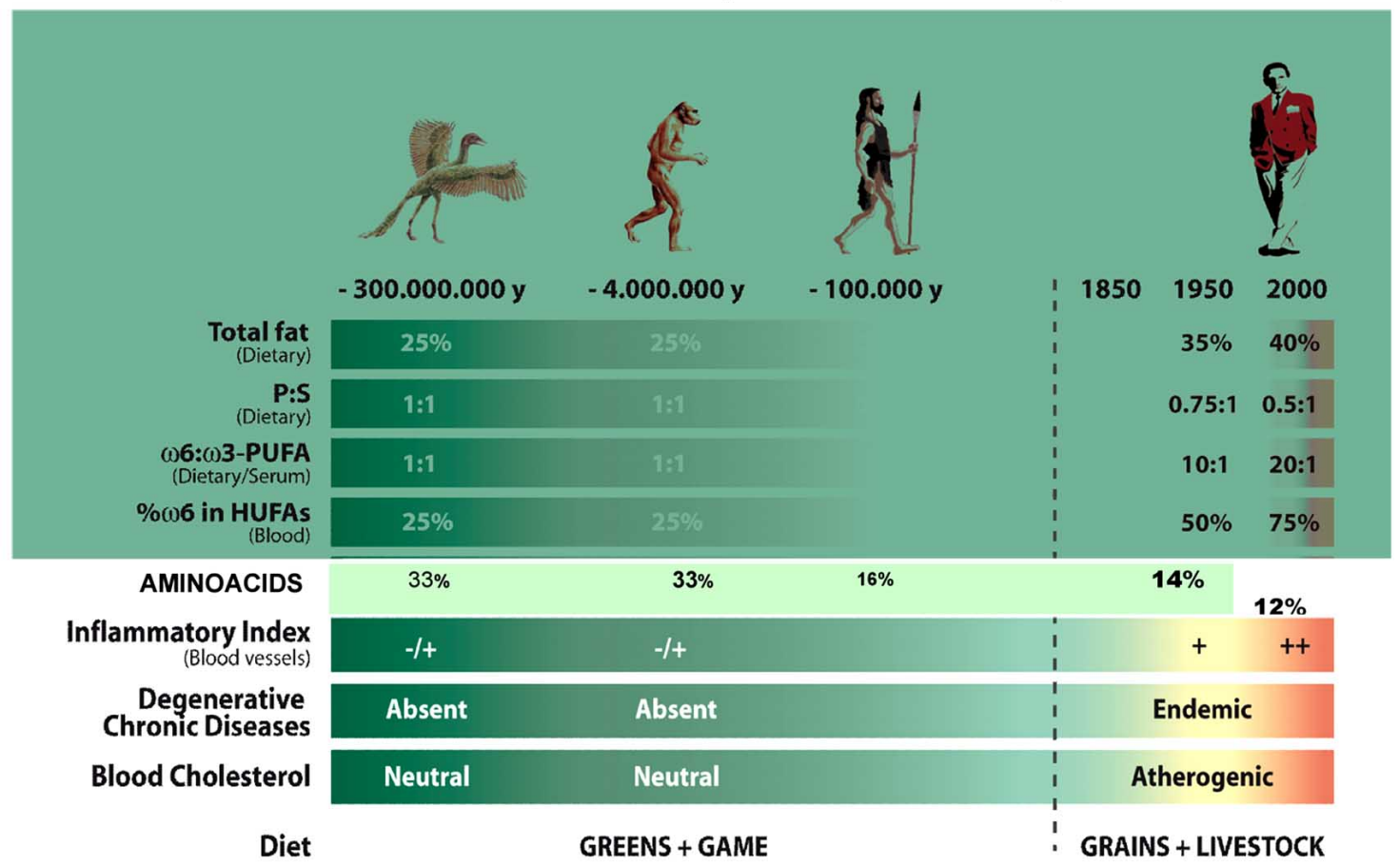

Fig. (1). Nutrient intake in Palaeolithic period, hunter-gatherers and modern man.

Tryptophan, a precursor of serotonin and melatonin, plays an important role in health and disease and its deficiency may underlie many types of brain disease such as quality of sleep and disturbance in sleep mediated by melatonin.

A recent study demonstrated the effect of the simultaneous dietary administration of fish protein and fish oil, two macronutrients found in fish meat, on cholesterol metabolism in rats [17]. Male Wistar rats were divided into four groups and fed an AIN-93G modified hypercholesterolemic diet with casein $(20 \%)+$ soybean oil $(7 \%)$, casein $(10 \%)+$ fish protein $(10 \%)+$ soybean oil $(7 \%)$, casein $(20 \%)+$ soybean oil $(5 \%)+$ fish oil $(2 \%)$, and casein $(10 \%)+$ fish protein $(10 \%)+$ soybean oil $(5 \%)+$ fish oil $(2 \%)$ for four weeks. Cholesterol metabolism was measured through serum and liver cholesterol assay, fecal cholesterol and bile acid excretion levels, and liver mRNA expression levels of enzymes and nuclear receptors involved in cholesterol homeostasis. Dietary fish protein decreased serum and liver cholesterol contents, perhaps through increasing fecal cholesterol and bile acid excretion and liver cholesterol -7-alphahydroxylase expression level. Dietary fish oil, on the other hand, decreased liver cholesterol content, perhaps due to the suppression of cholesterol synthesis through a decrease in the 3-hydroxy-3-methylglutaryl-coenzyme A reductase expression level; the serum cholesterol content was unchanged. This study found that the simultaneous dietary administration of fish protein and fish oil, which is achieved by the intake of intact fish muscle, has hypocholesterolemic effects that help prevent hyperlipidemia and atherosclerosis. In another study [20], the ratio of insulin-stimulated glucose versus amino acid clearance was decreased 5.4-fold in diabetic pigs, which was caused by a 3.6-fold decrease in glucose clearance and a 2.0-fold increase in non-essential amino acid clearance. In parallel with the Randle concept (glucose- fatty acid cycle), the present data suggest the existence of a glucose and non-essential amino acid substrate interaction in diabetic pigs whereby reduced insulin-stimulated glucose clearance seems to be partly compensated by an increase in non-essential amino acid clearance whereas essential amino acids are preferentially spared from an increase in clearance.

\section{METABOLISM OF AMINO ACIDS}

The plasma concentration of an amino acid is the result of its rates of appearance in and disappearance from plasma. As for most nutrients, amino acids rate of appearance and rate of disappearance are tightly regulated and at the postabsorptive state, The rate of appearance equals the rate of disappearance. Factors controlling rate of appearance are protein intake and tissue release; those controlling rate of disappearance are tissue uptake and body losses (urine, sweat, etc.). Regulation of plasma amino acids concentrations involves hormones, in particular insulin and glucagon, both of which induce hypoaminoacidemia (but for quite different reasons), and cortisol, which induces hyperaminoacidemia. In addition, in pathologic states, catecholamines, thyroid hormones, and cytokines modulate plasma amino acid levels. Peripheral availability of amino acids after protein ingestion is controlled by the liver, with an activation of ureagenesis in hyperprotein feeding and repression during a hypoprotein diet. The arginine-to-citrulline pathway in the intestine plays a key role in this adaptive process. In some circumstances tissue uptake of amino acids and further metabolism depend on plasma concentrations of the amino acids. Plasma glutamine level may be the driving force controlling the flux of this amino acid at the muscle level. Also, channelling of the arginine cellular pathways means that plasma arginine is a major controlling component of nitric oxide synthesis in endothelial and immune cells. All these features explain the 
excessive increase in glutamine and arginine demands, in particular for energy expenditure, leading to morbidity (e.g., gut atrophy, muscle wasting, and immune dysfunction) in stressed patients. Normoaminoacidemia is not synonymous with health because this state is observed in level 2 starvation when rate of appearance and rate of disappearance decrease, or after minor injury, the rates increase. Hyperaminoacidemia may be the consequence of organ failure (rate decreases) or excessive amino acid intake during parenteral nutrition (rate increases). Hypoaminoacidemia is observed after organ removal (rate decreases, e.g., decrease in citrulline concentration in short bowel syndrome) or in stress situations (rate increases). Mere determinations of plasma amino acid concentrations at the basal state (i.e., postabsorptive) provide rather limited information. Their usefulness can be improved by measuring arteriovenous differences or performing time course measurements, but techniques based on stable isotopes are necessary to obtain more precise information on the behavior of a particular amino acid or group of amino acids.

\section{GLUTAMATE AS PRECURSOR OF ARGININE}

L-Arginine is synthesised from glutamine, glutamate, and proline via the intestinal-renal axis in humans and most other mammals (including pigs, sheep and rats). Arginine degradation occurs via multiple pathways that are initiated by arginase, nitric-oxide synthase, arginine:glycine amidinotransferase, and arginine decarboxylase. These pathways produce nitric oxide, polyamines, proline, glutamate, creatine, and agmatine with each having enormous biological importance. Arginine is also required for the detoxification of ammonia, which is an extremely toxic substance for the central nervous system. There is compelling evidence that arginine regulates interorgan metabolism of energy substrates and the function of multiple organs. The results of both experimental and clinical studies indicate that arginine is a nutritionally essential amino acid for spermatogenesis, embryonic survival, fetal and neonatal growth, as well as maintenance of vascular tone and hemodynamics. Moreover, a growing body of evidence clearly indicates that dietary supplementation or intravenous administration of arginine is beneficial in improving reproductive, cardiovascular, pulmonary, renal, gastrointestinal, liver and immune functions, as well as facilitating wound healing, enhancing insulin sensitivity, and maintaining tissue integrity. Additionally, arginine or L-citrulline may provide novel and effective therapies for obesity, diabetes, and the metabolic syndrome. The effect of arginine in treating many developmental and health problems is unique among amino acids, and offers great promise for improved health and wellbeing of humans and animals.

\section{GLUTAMATE}

Glutamate, a derivative of the non-essential amino acid, L-glutamate, is the most abundant amino acid found in nature, and it is present, in a free and bound form, in many foods of animal and plant origin (Table 4). Food additives such as monosodium glutamate (MSG) or hydrolysed vegetable proteins are also significant sources. Glutamate is a major taste component of dietary protein, and its unique taste has been described as 'Umami' [6, 7]. Evidence from human subjects, rats and non-primates indicate that free glutamate functions as a signal to regulate protein intake and nutritional status, as well as other physiological processes, i.e. thermoregulation and energy homeostasis [8].

MSG, the sodium salt of glutamic acid, is a food additive used as a flavoring agent for enhancing taste. MSG intensifies flavour and also has the property to act as nutrient and salt substitute. It is frequently added to processed foods and shaken onto foods during preparation, particularly in Asian cuisine. The global market for fermentation products is expected to rise by $4.8 \%$ per year. Evaluations by the US Food and Drug Administration and other organizations concluded that MSG was a safe food ingredient for the general population. A potential explanation for the MSGobesity link is altered regulatory mechanisms that affect fat metabolism, which may be due to sedentary behaviour which is highly prevalent among populations in the developed countries.

After the discovery of sodium glutamate by Ritthausen in 1866, it was Kikunae Ikeda of Tokyo Imperial University in Japan who discovered its flavor- producing effect, and the commercial aspects of free glutamate were noticed by the food industry. He wanted to commercialize the component of kombu seaweed that produces umami taste as seasoning. Umami taste has been established as one of the five basic tastes, distinct from the other basic tastes such as saltiness, bitterness, sourness and sweetness. This taste possibly was known to ancient man because most of the evolutionary foods are rich in glutamate as given in Table $4[2,4,5]$. Behind the establishment of the new taste conception, scientific evidence for the physiological significance of free glutamatecontaining foods has accumulated over a century since its first discovery. Oral stimulation by free glutamate evokes the cephalic phase of food digestion, such as an induction of pancreatic juice secretion. In healthy and elderly volunteers, oral intake of free glutamate stimulates salivation which is essential for mastication and swallowing [6].

\section{INTERACTIONS OF AMINO ACIDS AND W-3 FATTY ACIDS}

The cell membranes of various cells, (e.g., endothelium, macrophages, platelets) are composed of amino acids and omega- 3 fatty acids. It is possible that fatty acids in conjunction with antioxidants, vitamins and minerals interact with amino acids in the synthesis and release of various enzymes and hormones. W-3 fatty acids (e.g., EPA and DHA) are incorporated into the phospholipids of the cell membranes of various cells which are made up of amino acids. It is important that all the cell membranes, mitochondria, DNA, RNA, enzymes hormones and neurotransmitters are made of amino acids. All the antioxidant enzymes (e.g., catalase, glutathione peroxidase, ceruloplasmin, and neurotransmitters; adrenaline, noradrenaline, serotonin cortisol, acetylecholine, prostaglandins as well as gut hormones; ghrelin, leptin, cholecystokinin, incretins, brain-derived neurotrophic factor and insulin) are made of amino acids. The majority of the magnesium and coenzyme Q10 present in the body are intracellular and present in the inner cell membrane of mitochondria. Deficiency of w-3 fatty acids and amino acids may cause dysfunction of cell membrane, resulting in increased susceptibility of these cells to enhance coagulation which predisposes athero-thrombosis. Endothelial cell damage due 
to deficiency of amino acids may be the first step, initiating the process, leading to increased activation of macrophages, platelets and red blood cells; causing atherogenesis and thrombosis.

\section{EFFECT OF GLUTAMATE ON BRAIN FUNCTION}

Gustatory and anticipatory cephalic stimuli during a meal yield nutritional information and aid efficient food digestion $[6,7,18-23]$. There is evidence that mammals, including humans, can detect the amount of dietary protein and its quality via cephalic relay to initiate proper digestion in the upper gastrointestinal tract. Apart from gustatory stimuli, visceral sensing by the abdominal vagus conveys primary afferent nutritional information from the digestive system to the brain. Further evidence showed that abdominal vagal afferents, which were innervated into the stomach and intestine sending information to the brain, were activated by luminal glutamate $[6,7]$. There is an existence of a glutamate signalling system involving metabotrophic glutamate receptors in the gastrointestinal tract $[6,7,23]$. Luminal glutamate in the stomach and intestine provides the efferent reflection of the abdominal vagus, supporting the modulation of exocrine and endocrine excretion during digestion. These results strongly indicate that glutamate has regulatory effects on the food digestive processes through the gut nutrient-sensing system. Glutamate plays physiological and nutritional roles and initiates digestion in the stomach as well as anticipating subsequent processes in the small intestine and the liver [2123]. The physiological significance of dietary free glutamate in the regulation of gut function, focuses on the visceral sensation from the stomach which appears to be very interesting.

Increased intake of salt $>5 \mathrm{~g} /$ day and excess of sugar may be associated with cardiovascular diseases and cancer. Increased consumption of sugar also increases the risk of obesity, metabolic syndrome, type 2 diabetes mellitus, gall bladder diseases, bone and joint diseases as well as degenerative diseases of the brain. Several experts advise on decreasing the intake of salt and sugar by substituting monosodium glutamate which makes the foods highly palatable and decreases the need for salt and sugar. In one study among 11 elderly Japanese, addition of monosodium glutamate to rice gruel for 2 months was associated with an improvement in nutritional status and quality of life as well as increase in lymphocyte count indicating better immunity [24]. No effect was noticed on mean body mass index, blood pressures, serum creatinine and liver enzymes. These findings indicate that glutamate may be supplemented and substituted for salt and sugar, to enhance palatability of foods.

\section{GLUTAMATE AND OBESITY}

Experimental studies indicate that monosodium glutamate (MSG) can induce hypothalamic lesions and leptin resistance, possibly influencing energy balance, leading to overweight. In an experiment on male Sprague Dawley rats, glutamate consumption suppressed weight gain, fat deposition and leptin levels [25]. The glutamate supplement was $1 \%$ MSG solution which actually showed weight gain reduction when given with high fat diet compared to no monosodium glutamate with high fat diet. In another experiment of 36 mice, MSG was administered with low and high fat diets and water with and without MSG [26]. After 16 weeks fol- low up, no effect was observed on weight gain and $1.0 \%$ MSG solution was greatly preferred over plain water. Mice fed low fat diets, having access to MSG showed a consistent preference.

The INTERMAP study included 752 Chinese, mean age $50 \mathrm{yrs}$, which is too small a number for a cross sectional survey [27]. Since MSG intake was positively associated with overweight in this study, it was publicized too much in the media. This study involving 752 healthy Chinese $(48.7 \%$ women), aged 40-59 years, randomly sampled from three rural villages in north and south China. The great majority of participants prepared their foods at home, without use of commercially processed foods. Diet was assessed with four in-depth multipass 24-h recalls. Participants were asked to demonstrate MSG amounts added in food preparation. Amounts shaken out were weighed by trained interviewers. Overweight was defined as BMI 25.0 or $23.0 \mathrm{~kg} / \mathrm{m}^{2}$ (based on World Health Organization recommendations for Asian populations). Eighty-two percent of participants were MSG users. Average intake was $0.33 \mathrm{~g} /$ day (s.d. = 0.40). With adjustment for potential confounders including physical activity and total energy intake, MSG intake was positively related to BMI. For users in the highest tertile of MSG intake compared to nonusers, the multivariable-adjusted odds ratios of overweight (BMI 23.0 and 25.0) were $2.10 \quad(95 \%$ confidence interval, 1.13-3.90, $\mathrm{P}$ for trend across four MSG categories $=0.03)$ and $2.75(95 \%$ confidence interval, 1.28$5.95, \mathrm{P}=0.04)$. This research provides data that MSG intake may be associated with increased risk of overweight independent of physical activity and total energy intake in humans. In this study, our ability to examine the relationship of MSG intake and obesity was limited by the small number of persons $(3 \%)$ with BMI 30.0. However, the partial correlation between MSG intake and BMI approached significance. Hence it is reasonable to infer that MSG intake is also associated with higher prevalence of obesity. This study is also limited by lack of data on leptin and adipsin concentrations. In addition, the INTERMAP findings are cross-sectional, but nevertheless unique; this topic has not been previously pursued in human population samples. Thus this small cross-sectional study of 752 healthy Chinese men and women suggest that monosodium glutamate (MSG) may be associated with overweight/obesity, and these findings raise public concern over the use of MSG as a flavour enhancer in many commercial foods. However, there are many limitations in the methodology of this study. Prevalence of overweight was significantly higher in MSG users than nonusers, which may be because they eat possibly, more food and are less physically active because assessment of occupational physical activity is open to bias. The other important weakness was that the sample size was too small $(n=752)$. Dietary intakes were obtained by 24-hour recall which is open to bias and mistakes in the assessment. We need to assess dietary intake by 7-days food intake dietary diaries or by weighing of foods for 7 days for finding out accurate intake of foods. The MSG intake can vary around the 7 week days. Cross-sectional surveys are open to bias by the experts recording the data and hence a prospective cohort study is necessary to find out the association of MSG intake with obesity.

In a more recent larger study, dietary intakes of glutamate were studied among approx.1300 Chinese, mean age 55 
years, by food frequency questionnaire [28]. The aim of this analysis was to investigate a possible association between MSG intake and obesity, and determine whether a greater MSG intake is associated with a clinically significant weight gain over 5 years. Data from 1282 Chinese men and women who participated in the Jiangsu Nutrition Study were analysed. MSG intake and body weight were quantitatively assessed in 2002 and followed up in 2007. MSG intake was not associated with significant weight gain after adjusting for age, sex, multiple lifestyle factors and energy intake. When total glutamate intake was added to the model, an inverse association between MSG intake and $5 \%$ weight gain was found $(P=0 \cdot 028)$, but when the model was adjusted for either rice intake or food patterns, this association was abolished. These findings indicate that when other food items or dietary patterns are accounted for, no association exists between MSG intake and weight gain.

Further studies are necessary to find out the beneficial effects of glutamate on various gut hormones and other body systems.

The food that we eat can directly regulate gene expression in our body. According to a recent study, microRNAs from common plant crops such as rice and cabbage can be found in the blood and tissues of humans and other plant-eating mammals [29]. One microRNA in particular, MIR168a, which is highly enriched in rice, was found to inhibit a protein that helps to remove low-density lipoprotein (LDL) from the blood, suggesting that microRNAs can influence gene expression across kingdoms. This study further confirms the role of dietary amino acids in biological functions in our body systems.

In brief, the balance of omega-6/omega-3 fatty acids as well as amino acids is an important determinant in decreasing the risk for central obesity, type 2 diabetes mellitus, coronary artery disease (CAD), both in the primary and secondary prevention studies [9-16]. Increased dietary intake of linoleic acid leads to oxidation of LDL, platelet aggregation, and interferes with the incorporation of EPA and DHA into cell membrane phospholipids and a decrease in HDL which may worsen in presence of a deficiency of amino acids. Both omega- 6 and omega- 3 fatty acids influence gene expression which may be modulated by amino acids. EPA and DHA have the most potent anti-inflammatory effects that may be enhanced by amino acids. Inflammation is at the base of many chronic diseases, including coronary heart disease, diabetes, arthritis, cancer, osteoporosis, mental health, dry eye disease and macular degeneration [10-15]. Dietary intake of omega- 3 fatty acids and amino acids may prevent the development of disease, particularly in persons with genetic variation, as for example in individuals with genetic variants at the 5-LO (lipoxygenase) pathway and the development of CAD. The omega-3 fatty acids from dietary sources or through supplementation are associated with a reduced risk of impaired cognitive function, dementia and improve cognitive development, mood state, increase vigor and a sense of well-being, suggesting a direct action of omega-3 fatty acids on the CNS. Similar actions have been observed after supplementation with amino acids. CVDs are multigenic and multifactorial [9-16]. The dietary intake and plasma levels or red cell membrane phospholipids of w-3 fatty acids and amino acids should be determined before and after the intervention study to demonstrate their potential role in the prevention of diseases. The role of amino acids in the diet rich in w-3 fatty acids could be additive.

\section{ACKNOWLEDGEMENT}

Acknowledgements are due to International College of Nutrition for providing the logistic support to write this article.

\section{CONFLICT OF INTEREST}

None declared.

\section{REFERENCES}

[1] De Meester F. Progress in lipid nutrition. In: Simopoulos AP, De Meester F, Eds. A balanced omega-6/omega-3 fatty acid ratio. Cholesterol and Coronary Heart Disease. World Rev Nutr Diet Basel Karger 2009; 100:110-21.

[2] Simopoulos AP. Evolutionary aspects of the dietary omega6/omega-3 fatty acid ratio: medical implications. In Simopoulos AP, De Meester F, Eds. A balanced omega-6/omega-3 fatty acid ratio. Cholesterol and Coronary Heart Disease. World Rev Nutr Diet Basel Karger 2009; 100:1-21.

[3] Eaton SB, Konner M, Shostak M. Stone agers in the fast lane: chronic degenerative diseases in evolutionary perspective. Am J Med 1988; 84: 739-49.

[4] Eaton SB, Eaton SB III, Sinclair AJ, Cordain L, Mann NJ. Dietary intake of long chain polyunsaturated fatty acids during the Palaeolithic period. World Rev Nutr Diet 1998; 83: 12-23.

[5] Wu G. Amino acids metabolism functions and nutrition. Amino Acids 2009; 37: 1-17.

[6] Uneyama H, San Gabriel A, Kawai M, Tomoe M, Torii K. Physiological role of dietary free glutamate in the food digestion. Asia Pac J Clin Nutr 2008; 17: 372-5.

[7] McCabe C, Rolls ET. Umami: a delicious flavor formed by convergence of taste and olfactory pathways in the human brain. Eur J Neurosci 2007; 25: 1855-64.

[8] Avshalumov MV, Rise ME. Activation of ATP-sensitive K+ (KATP) channels by $\mathrm{H}_{2} \mathrm{O}_{2}$ underlies glutamate-dependent inhibition of striatal dopamine release. Proc Natl Acad Sci USA 2003; 100: 11729-34.

[9] Singh RB, De Meester F, Wilczynska A, Wilson DW, Hungin APS. The liver-pancreas and brain connection in the pathogenesis of obesity and diabetes mellitus. World Heart J 2011, (in press).

[10] Singh RB, De Meester F, Wilczynska A. The Tsim Tsoum approaches for prevention of cardiovascular diseases. Cardiol Res Prac 2010; 2010: 824938 .

[11] Lorgeril M, Renaud S, Mamelle N, et al. Mediterranean alphalinolenic acid-rich diet in secondary prevention of coronary heart disease. Lancet 1994; 343(8911): 1454-9. [Erratum in: Lancet 1995; 345(8951): 738].

[12] Renaud S, de Lorgeril M, Delaye J, et al. Cretan Mediterranean diet for prevention of coronary heart disease. Am J Clin Nutr 1995; 61(Suppl 6): 1360S-7S.

[13] Singh RB, De Meester F, Pella D, Basu TK, Watson R. Globalization of dietary wild foods protect against cardiovascular disease and all cause mortalities? A Scientific statement from the International College of Cardiology, Columbus Paradigm Institute and the International College of Nutrition. Open Nutra J 2009; 2: 42-5.

[14] Singh RB, Dubnov G, Niaz MA, et al. Effect of an IndoMediterranean diet on progression of coronary disease in high risk patients: a randomized single blind trial. Lancet 2002; 360: 1455-61.

[15] Trichopoulou A, Bamia C, Trichopoulos D. Anatomy of health effects of Mediterranean diets. Greek epic prospective heart study. BMJ 2009; 338: b2337.

[16] Frieden TR, Berwick DM. The million's heart initiative- preventing heart attacks and strokes. N Engl J Med 2011; 365: e27.

[17] Hosomi R, Fukunaga K, Arai H, Kanda S, Nishiyama T, Yoshida M. Effect of simultaneous intake of fish protein and fish oil on 
cholesterol metabolism in rats fed high cholesterol diets. Open Nutra J 2011; 4: 12-9.

[18] Young VR, Ajami AM. Glutamate: an amino acid of particular distinction. J Nutr 2000; 130: 892S-900S.

[19] Tomporowski PD. Effects of acute bouts of exercise on cognition. Acta Psychologica 2009; 112: 297-324.

[20] Koopmans SJ, VanderMeulen J, Wijdenes J, Corbijn H, Dekker R. The existence of an insulin stimulated glucose and non-essential but not essential amino acid substrate interaction in diabetic pigs. BMC Biochem 2011; 23(12): 25.

[21] Uematsu A, Tsurugizawa T, Kondoh T, Torii K. Conditioned flavor preference learning by intragastric administration of Lglutamate in rats. Neurosci Lett 2009; 451: 190-3.

[22] Uematsu A, Tsurugizawa T, Uneyama H, Torii K. Brain-gut communication via vagus nerve modulates conditioned flavor preference. Eur J Neurosci 2010; 31: 1136-43.

[23] Kitamura A, Tsurugizawa, Torii K. Biological significance of glutamate signalling during digestion of food through the gut-brain axis. Digestion 2011; 83(Supple 1): 37-43.
[24] Toyama K, Tomoe M, Inoue Y, Sanbe A, Yamamoto Y. A possible application of monosodium glutamate to nutritional care for elderly people. Biol Pharmacol Bull 2008; 31: 1852-4.

[25] Kondoh T, Torii K. MSG intake suppresses weight gain, fat deposition, and plasma leptin levels in male sprague dawley rats. Physiol Behav 2008; 93: 135-44.

[26] Ren X, Ferreira JG, Yecket CW, Kondoh T, de Araujo IE. Effects of ad libitum ingestion of monosodium glutamate on weight gain in C57BL6/J mice. Digestion 2011; 83(Supple 1): 32-6.

[27] He Ka, Zhao L, Deviglus ML, et al. Association of monosodium glutamate with overweight in Chinese adults: The INTERMAP study. Obesity 2008; 16: 1875-8.

[28] Shi Z, Luscombe MND, Wittert GA, et al. Monosodium glutamate is not associated with obesity or greater prevalence of weight gain over 5 years: Findings from the Jiangsu Nutrition Study of Chinese adults. Brit J Nutr 2010; 104: 457-63.

[29] Zhang L, Hou D, Chen X, et al. Exogenous plant MIR168a specifically targets mammalian LDLRAP1: evidence of cross-kingdom regulation by microRNA. Cell Res 2011. [Epub ahead of print]

(c) Takahashi et al.; Licensee Bentham Open.

This is an open access article licensed under the terms of the Creative Commons Attribution Non-Commercial License (http://creativecommons. org/licenses/ by-nc/3. 0/) which permits unrestricted, non-commercial use, distribution and reproduction in any medium, provided the work is properly cited. 